\title{
Skin proteomics - analysis of the extracellular matrix in health and disease
}

\author{
Jörn Dengjel ${ }^{\mathrm{a}}$, Leena Bruckner-Tuderman ${ }^{\mathrm{b}}$ and Alexander Nyström ${ }^{\mathrm{b}}$ \\ aDepartment of Biology, University of Fribourg, Fribourg, Switzerland; ${ }^{b}$ Department of Dermatology, Faculty of Medicine, Medical Center - \\ University of Freiburg, Freiburg, University of Freiburg, Freiburg, Germany Germany
}

\begin{abstract}
Introduction: The skin protects the human body from external insults and regulates water and temperature homeostasis. A highly developed extracellular matrix (ECM) supports the skin and instructs its cell functions. Reduced functionality of the ECM is often associated with skin diseases that cause physical impairment and also have implications on social interactions and quality of life of affected individuals.

Areas covered: With a focus on the skin ECM we discuss how mass spectrometry (MS)-based proteomic approaches first contributed to establishing skin protein inventories and then facilitated elucidation of molecular functions and disease mechanisms.

Expert opinion: MS-based proteomic approaches have significantly contributed to our understanding of skin pathophysiology, but also revealed the challenges in assessing the skin ECM. The numerous posttranslational modifications of ECM proteins, like glycosylation, crosslinking, oxidation, and proteolytic maturation in disease settings can be difficult to tackle and remain understudied. Increased ease of handling of LC-MS/MS systems and automated/streamlined data analysis pipelines together with the accompanying increased usage of LC-MS/MS approaches will ensure that in the coming years MS-based proteomic approaches will continue to play a vital part in skin disease research. They will facilitate the elucidation of molecular disease mechanisms and, ultimately, identification of new druggable targets.
\end{abstract}

KEYWORDS

Skin fragility; wound healing; mass spectrometry

\section{Introduction}

The skin is the largest organ of the human body, with an area of ca. $1.7 \mathrm{~m}^{2}$ and a weight of ca. $3 \mathrm{~kg}$. It consists of three different layers - epidermis, dermis, and subcutis - that have distinct tissue architectures and functions. Through tight cohesion to each other and through highly regulated interactions, the three layers form a barrier to the environment ensuring defense against microbial attacks and chemical and physical influences from the environment, protection against loss of water or proteins, and temperature control, which is essential for the proper functionality of the entire organism [1]. Loss of its protective functions is associated with numerous genetic and acquired skin diseases that manifest with a broad spectrum of symptoms. It may not be well known that about $20 \%$ of patients seeking help of a general practitioner have skin symptoms [2]. Therefore, not only the medical but also the socio-economic importance of understanding the physiological functions and interactions of skin cells and their derailment in disease states is huge.

Unbiased, i.e. hypothesis-free, global proteomic approaches have offered unprecedented opportunities to disclose molecular players in skin homeostasis and pathology. This review deals with MS-based proteomic analyses of the skin and skin cells, keratinocytes and fibroblasts, and covers historic publications as well as recent work focusing on molecular mechanisms. A special focus is the skin extracellular matrix (ECM). We highlight examples of how MS-based proteomic analyses have generated important new information on skin diseases and delivered novel therapeutic cues, especially for skin fragility.

The ECM is essential to the skin. It aids the formation of an effective, protective barrier and provides toughness to shearing forces, whilst at the same time allowing sufficient elasticity to not restrict movement. The creation of an ECM scaffold to support a tissue encompassing such seemingly incompatible abilities is achieved through the close interactions of multiple, highly specialized substructures, which condition the skin with their unique properties. These substructures appear distinct in their ECM proteome and/or in their supramolecular arrangement and makeup four distinctive intertwined ECMs: the epidermal ECM, the dermalepidermal junction zone, the papillary dermal ECM, and the reticular dermal matrix. Assembly of these ECMs requires highly coordinated cell-cell and cell-microenvironment communications, in time and in space [3]. Within each ECM considerable heterogeneity exists that provides important biological functions, for example, by creating stem cell-supporting microniches [4].

The complex structure of the skin ECM and certain traits of it make its detailed proteomic analysis challenging. The ECM is made up of proteins that often need to undergo extensive posttranslational modifications to become functional, including hydroxylation of selected proline and lysine residues (elastin and collagens), intramolecular cross-linking, N- and O-linked glycosylation (glycoproteins and proteoglycans, respectively) and extracellular proteolytic maturation. The 
Article highlights

- Skin functions are dependent on a highly complex extracellular matrix (ECM).

- Both keratinocytes and skin fibroblasts contribute to the ECM.

- MS-based proteomic approaches yield unprecedented insights into the composition and regulation of the proteome of skin and skin cells.

- MS-based proteomic analyses of PTM and crosslinked ECM proteins are challenging and require special protocols

- Current robust MS-based proteomic analyses help identify new druggable targets in skin diseases.

ECM backbone is composed of large proteins such as collagens, non-collagenous glycoproteins including laminins, fibrillins and fibronectin, and proteoglycans with repetitive domains that are closely or more distantly homologous to each other and other ECM proteins [5-9]. Many proteins have low solubility, which is further reduced through intermolecular crosslinking by e.g. transglutaminases and lysyl oxidases [10]. However, for its proper organization and functionality the skin ECM is also dependent on smaller soluble proteins. A further challenge to achieve comprehensive coverage and quantitative assessment of the skin by proteomic approaches comes from the great range in protein abundances. To illustrate this, there is a more than 70,000 -fold difference in abundance of collagen I and collagen VII in the skin - yet both proteins are essential for skin stability [11].

\section{The skin: structure, major cell types, and the organization of its ECM proteome}

The main layers of the skin, the epidermis, the dermis, and the subcutis (Figure 1) act synergistically to form a functional organ.
However, they are distinctly disparate in terms of cellular composition and ECM organization. The following paragraphs will briefly summarize the cellular and extracellular proteome characteristics of the skin layers. The aim is to provide the readers with information to follow the subsequent discussion of the use of MS-based proteomic approaches for the understanding of ECM function in skin pathobiology. For a more detailed review of the skin ECM, we refer readers to our recent reviews on this subject [12-14].

\subsection{The epidermis and epidermal keratinocytes}

The outermost layer, the epidermis, is a multi-level cornified epithelium and a tightly packed cellular compartment with little intercellular space or ECM. The epidermis consists of $90 \%$ of keratinocytes, but contains also minor cell populations, such as melanocytes, Langerhans cells, and Merkel cells that have specific functions in pigment production, first-line immune defense, and in touch sensation, respectively [1]. Keratinocytes secure epidermal integrity and the formation of the skin barrier. The basal keratinocytes in the lowermost epidermis, the stem cell compartment of the epidermis, sit on a basement membrane, and undergo vertical proliferation and differentiation cycles that ensure the renewal of the epidermis every 30 days. During this process, as they move from the basal layer of the epidermis toward its surface, the keratinocytes differentiate first into spinous cells and then to granular cells. Each of the differentiation stages exhibits a specific expression profile of intermediate filament keratins and barrier proteins, such as loricrin or filaggrin. Finally, the terminally differentiated keratinocytes undergo programmed cell death and contribute with their plasma membranes, cytoskeleton, structural proteins, extra- and intracellular lipids to the formation of the horny layer (stratum corneum) that is constantly being sloughed off [15]. Thus, the epidermal cells

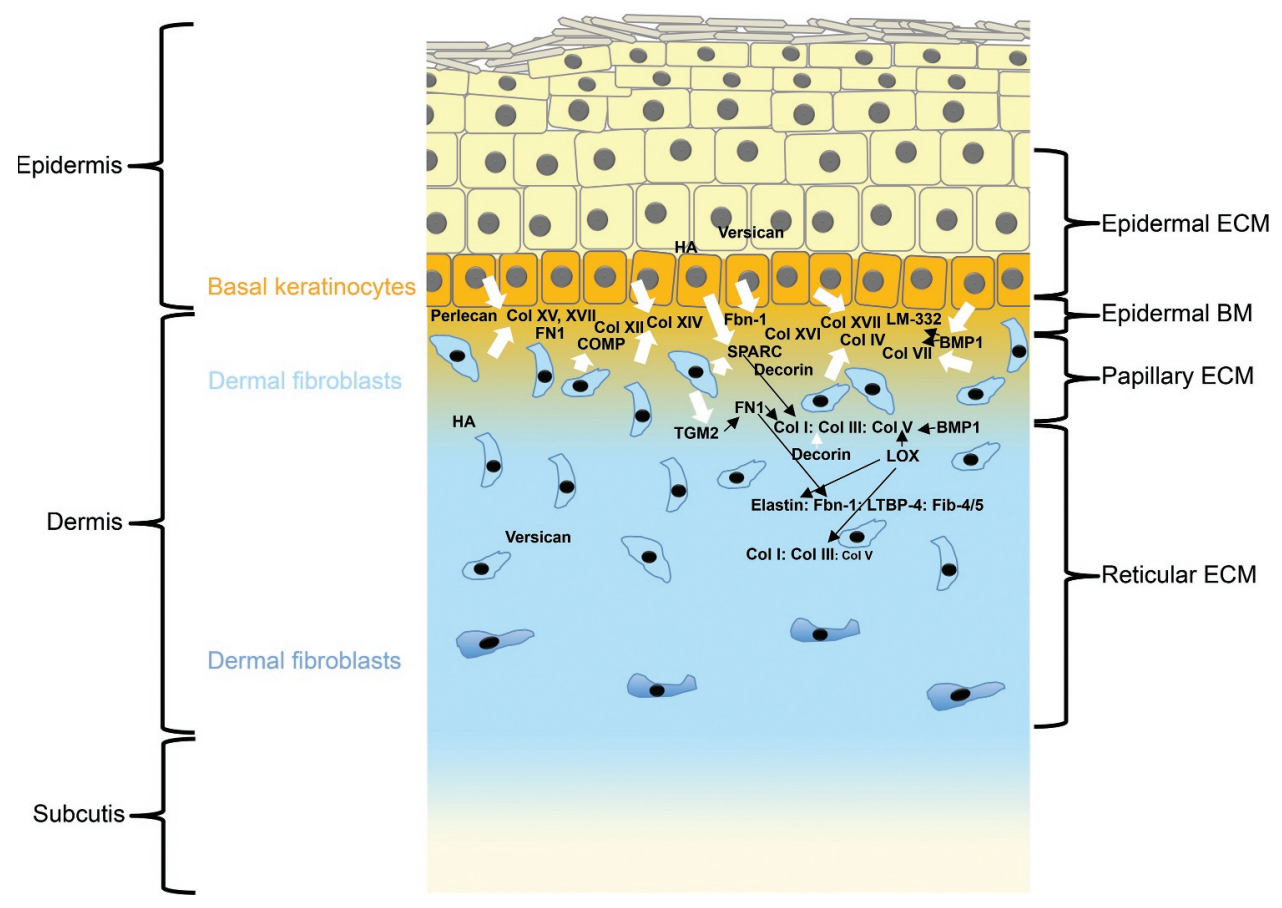

Figure 1. Skin structure and ECM crosstalk. Schematic illustration of skin indicating tissue compartments and the different skin ECMs. In addition, selected key ECM proteins with their predominant location and main producing cells are shown with white arrows. Essential protein-protein interactions are shown with black arrows. 
represent a dynamic system where basal keratinocytes replenish the cellular components of the entire epidermis and the building blocks for the skin barrier via a differentiation process [15].

Parallel to this protective function as a skin barrier, epidermal keratinocytes exert significant regulatory functions [16]. They instruct other epidermal cells, but also signal into the dermis and regulate cellular events there via paracrine mechanisms. For example, upon epidermal damage, ample interleukin 1 is secreted into the dermis where it induces inflammatory processes and subsequent repair [16].

\subsection{The epidermal ECM}

The epidermis is an ECM-poor tissue compartment (Table 1); however, the scant ECM is essential for the formation and maintenance of a functional epidermal microenvironment and finally contributes to producing the skin barrier. Activity of extracellular proteinases of the kallikrein family contributes to this process [17]. The extracellular microenvironment varies in its composition from the inner to the outer layers of the epidermis. In the upper granular and horny layers, the environment is lipophilic; deposition of lipids contributes to the impermeability of the skin $[18,19]$. In extracellular spaces around the living epidermal layers composed of basal and spinous keratinocytes, the environment is hydrophilic with a notable presence of glycosaminoglycans (GAGs) such as hyaluronan and cell-surface, pericellular, and extracellular proteoglycans such as syndecan 1, perlecan, and versican (Figure 1) [19-22].

\subsection{The dermal-epidermal junction zone}

The dermal-epidermal junction zone (DEJZ) binds and separates the epidermis and the dermis. A firm anchorage of these layers is important for resistance of the skin to frictional challenges. Structurally, the DEJZ is a highly specialized, extended basement membrane zone that is often described in ultrastructural terms. As viewed in the electron microscope, it consists of several suprastructural units: hemidesmosomes at the inner plasma membrane of basal keratinocytes, the anchoring filaments, the bi-layered basement membrane proper (with an electron-lucid lamina lucida and an electron-dense lamina densa), and the anchoring fibrils that protrude from the lamina densa into the uppermost dermis [23]. For practical reasons, the scientific community continues to use these ultrastructural terms, although it is unlikely that the functional supramolecular units in vivo will strictly correspond to the images seen in the electron microscope. Through specific protein-protein interactions the molecular components of these suprastructural units provide a cohesive 'chain' that extends from inside the basal keratinocytes into the dermal ECM and thus enforces dermalepidermal anchorage. The molecular components of the DEJZ are introduced below and in Table 1.

\subsection{The cell-surface and ECM proteome of the DEJZ}

Under steady-state conditions, the main adhesion structures of the basal keratinocytes are the hemidesmosomes and the anchoring filaments. These contain a number of molecules, including cytoskeleton linker proteins, integrins, transmembrane collagens, and ECM components that undergo highly specific protein-protein interactions. At the molecular level, the keratin cytoskeleton in basal keratinocytes is linked via the P1a isoform of plectin and BP230 to the intracellular tail of integrin $a 6 \beta 4$, which, in turn, binds also the intracellular domain of transmembrane collagen XVII and tetraspanin CD151. This complex condenses into the hemidesmosome [24]. The extracellular domains of integrin a6 $\beta 4$ and collagen XVII support adhesion by interacting with laminin-332 in the basement membrane. Collagen XVII further promotes adhesion through binding to the basement membrane collagen IV in the lamina densa (Figure 1) [25]. The quantitatively minor transmembrane collagens XIII, XXII, and XXIII are also observed at the DEJZ and may support keratinocyte attachment to the epidermal basement membrane in certain contexts [26-29]. They exist in two forms that may have altered biological activity, a full-length transmembrane form, and a shed ectodomain that is released by ADAM- (A disintegrin and metalloproteinases) or furin-mediated shedding and incorporated in the pericellular ECM [30-32].

Keratinocytes utilize also other adhesive structures including integrin $\alpha 3 \beta 1$-based focal adhesions and integrin $\alpha 3 \beta 1$ - and a6 $\beta 4$-containing tetraspanin-enriched microdomains [33]. For both integrins laminins are the main ligands. Laminins are trimeric proteins composed of genetically distinct $\alpha, \beta$, and $\gamma$ chains

Table 1. The proteome and the glycome of the skin ECM.

\begin{tabular}{|c|c|}
\hline \multirow{4}{*}{ Epidermal ECM } & Selected key extracellular proteins and GAGs \\
\hline & Proteases: Kallikreins \\
\hline & Proteoglycans: Syndecan 1, perlecan and versican \\
\hline & Collagens: Collagen XIII, XVII, XXII and XXIII \\
\hline & Hyaluronan \\
\hline \multirow{6}{*}{ Epidermal basement membrane } & Proteases: BMP1 and BMP1-like proteinases \\
\hline & Laminins: Laminin-332 and -511 \\
\hline & Nidogen 1 and 2 \\
\hline & Proteoglycans: Perlecan, PRELP, collagen XV, collagen XVIII and versican \\
\hline & Collagens: Collagen IV, VII, XV, XVII and XVIII \\
\hline & Fibronectin \\
\hline \multirow[t]{6}{*}{ Papillary and reticular dermal ECM } & Proteases: BMP1 and BMP1-like proteinases \\
\hline & Enzymes: LOX and TGM2 \\
\hline & Matricellular proteins: COMP, SPARC, thrombospondin-1, periostin, tenascin $\mathrm{C}$ and $\mathrm{X}$ \\
\hline & Proteoglycans: Decorin, versican, biglycan, fibromodulin and lumican \\
\hline & Collagens: Collagen I, III, V, VI, XII, XIV and XV; \\
\hline & $\begin{array}{l}\text { Fibrillin microfibrils and elastic fiber proteins: Fibrillin-1 and -2, elastin, EMLIN-1 and-2, LTBP-4, fibulin- } 4 \text { and }-5 \\
\text { Hyaluronan }\end{array}$ \\
\hline
\end{tabular}


and named according to the chain composition, i.e. laminin-332 is made of laminin $\alpha 3, \beta 3$, and $\gamma 2$ chains [34]. Laminin-332 and laminin-511 are the major laminin isoforms at the DEJZ and both are mainly contributed by keratinocytes [35]; however, they are not equally distributed within the epidermal basement membrane. Laminin-511 is enriched around hair follicles, whereas laminin-332 is more abundant under the interfollicular epidermis (Figure 1). The laminin-511:laminin-332 ratio has been shown to regulate stem cell activity [36].

There are key structural differences between laminin-511 and laminin-332. Laminin-511 has three N-terminal short-arms, which are the parts of the laminin chains that are not folded into an a-helical coiled-coil with other chains [34]. Through intermolecular interactions of the short-arms laminin-511 can form a laminin network [37]. Contrastingly, laminin-332's short-arms are truncated and cannot participate in network formation [34]. However, although the usual gene product of the LAMA3 gene, the laminin a3 chain, is network incompetent, the gene may still potentially influence laminin network assembly through the expression of its alternative splice product, the LaNt a31 protein [38]. LaNt a31 is homologous to netrins and based on this has been proposed to regulate laminin network formation and/or stability and consequently basement membrane formation [38,39].

Subjacent to the hemidesmosomes, laminin-332, and the ectodomain of collagen XVII arrange into molecularly densely packed anchoring filaments [40], which connect to collagen VII-composed anchoring fibrils [41]. Within the basement membrane proper, perlecan, nidogen 1 and 2, and collagen VII link the laminin network to a collagen IV network. This consists mainly of collagen IV a1.a1.a2 but with a notable presence of collagen IV a5.a5.a6 [37,42,43] and provides stiffness and stability to the basement membrane [44]. Hybrid collagen/proteoglycans, collagen $\mathrm{XV}$, and collagen XVIII, are also present at the DEJZ $[45,46]$, but their functional contributions need further elucidation. Both keratinocytes and dermal fibroblasts are capable of expressing these collagens; however, collagen XV appears to be primarily a fibroblast product [4648]. PRELP is an extracellular proteoglycan at the DEJZ [49]. It belongs to small leucine-rich proteoglycans (SLRPs) [22] and has been proposed to function as an anchor between perlecan and collagen-containing fibrils in the papillary dermal ECM (Figure 1) [49]. Cultured keratinocytes have been shown to express another SLRP, decorin, but the physiological relevance of this remains to be explored $[48,50]$.

Proteolytic activity of the astacin-like proteinase BMP-1/ tolloid-like is essential for the assembly of a functional DEJZ, papillary, and reticular dermal ECM. These proteinases process proforms of multiple ECM components to mature functional molecules, including the SLRP biglycan, the N-terminal end of the laminin $\gamma 2$ chain, and the C-terminal end of laminin a3 in laminin-332, procollagen VII, and the C-terminal ends of procollagens I and III [51-53]. In addition, the other members of the astacin-like proteinases - meprin $a$ and $\beta$ - have been shown to be involved in processing of the $\mathrm{N}$ - and C-terminal ends of fibrillar procollagens in the skin [54]. ADAMTS2, -3 , and -13 are generally considered the main proteases involved in removal of the procollagen N-pro-termini [55]. Although often considered to be ECM degrading proteases, also matrix metalloproteinases (MMPs) are involved in the assembly of the DEJZ through e.g. processing of laminin-332 [56].

In healthy homeostatic skin, the glycoprotein fibronectin is present around the DEJZ and in the papillary dermis [57]. It can be synthesized at substantial levels by both keratinocytes and fibroblasts [48,58]. After an injury it is dramatically increased by deposition of plasma fibronectin and later by fibronectin produced by tissue-resident cells [59]. Fibronectin fills key roles during development and regeneration serving as an adhesive substrate for migrating cells but also as an important facilitator of fibrillar collagen and fibrillin microfibril deposition [60,61].

\subsection{The dermis and dermal fibroblasts}

The second important skin layer is the dermis (Figure 1). It provides tensile strength, elasticity, and resilience to the skin. Histologically the dermis can be divided into a thin superficial layer, the papillary dermis, and a thicker deeper layer, the reticular dermis. Although adnexal, vascular, lymphatic and neural structures traverse the dermis, fibroblasts constitute the major cell population embedded in the dermal connective tissue. These are ECM producing mesenchymal cells responsible for maintenance, repair, and regeneration of the dermis. Recent research data indicate that the superficial, middle, and deeper dermal layers harbor distinct fibroblast subpopulations [62], nevertheless definitive cell markers still remain elusive and functional characteristics that distinguish them are not fully understood. Quantitatively minor and changing cell populations in the dermis encompass immune cells. Upon need, they migrate into the skin and exert decisive functions in immune response, inflammation, and regenerative processes [63].

Apart from their structure-maintaining role, fibroblasts have regulatory functions. They can direct cell behavior in both the epidermis and the dermis, e.g. via paracrine signaling [64]. Intriguingly, also the dermal ECM itself has cell-instructive capabilities; it sequesters growth factors, cytokines, and other signaling molecules via specific binding to structural macromolecules [12]. A major regulatory mechanism is the release of these soluble factors, e.g. via proteolysis of ECM proteins or conformational changes that eliminate or weaken binding sites. The soluble molecules then migrate into the tissue microenvironment and influence cell functions.

These processes and mechanisms play an important role in epidermal-dermal cross-talk. The skin layers are in constant exchange, and cells sense changes in other compartments. Typical examples are physiological situations like development, repair, and regeneration after injury [63]. Examples of pathological conditions with highly active cross-talk between the epidermis and the dermis include skin fragility syndromes, perturbed wound healing, or the interactions between epithelial cancers and their stroma $[62,65]$.

\subsection{The papillary and reticular dermal ECM}

Composition and arrangement of the dermal ECM mirror that of the histological division into a papillary and a reticular layer with their specific tissue architectures and specialized ECMs (Table 1). The structural scaffold of the ECM is provided by 
collagen-, elastin- and microfibrils-containing supramolecular fibril networks, with a variety of associated distinct ECM components. These are often quantitatively minor ECM molecules, but they confer substantial effects on the suprastructures and provide specific ligand binding capabilities to the fibril networks. The interfibrillar space is filled with glycoproteins, proteoglycans, and ample hyaluronan with a very high waterbinding capacity that provides the skin with its tautness [12].

The papillary ECM just below the DEJZ is an extension of this zone providing additional points of stability and securing epidermal attachment to the dermis. This structure has higher flexibility than the more rigid epidermal basement membrane enabling deformable joining of the basement membrane and the reticular ECM [12]. As for the epidermal basement membrane, both keratinocytes and fibroblasts can contribute to its proteome. Collagen VI, predominantly collagen VI a1.a2.a3, assembles into a tissue-stabilizing microfibrillar network below the basement membrane [66]. Collagen $\mathrm{V}$ also shows notable accumulation below the epidermal basement membrane [67], where it can provide further anchoring stability by interacting with collagens IV and VI (Figure 1) [68].

The matricellular protein COMP (cartilage oligomeric matrix protein) can also be involved in the assembly of the papillary ECM by binding the FACIT (Fibril Associated Collagens with Interrupted Triple helices) collagen XII and collagen XIV [69]. Both of these collagens are expressed by dermal fibroblasts and epidermal keratinocytes $[47,48]$.

Fibrillin microfibrils connecting to the epidermal basement membrane-papillary ECM interface provide additional stability [70]. Although generally considered a fibroblastic product, interestingly, keratinocytes can also express fibrillin- 1 and 2 and assemble microfibrils in vitro [71,72]. Fibrillin- 1 is most abundant in adult tissue and fibrillin-2 is expressed during development and regeneration [73]. Additional molecules such as elastin microfibril interface-located proteins (EMILINs) 1 and 2 associate with dermal fibrillin microfibrils [74,75]. Collagen XVI is expressed by both keratinocytes and fibroblasts and colocalizes with fibrillin microfibrils at the epidermal basement membrane-papillary ECM interface, whilst also showing close proximity to collagen VII (Figure 1). Collectively, this suggests a role for it in supporting dermal-epidermal cohesion [76].

The matricellular protein tenascin- $X$ interacts with many proteins in the papillary ECM, including collagen XII, XIV, and fibrillar collagens. The latter fill key architectural functions [77,78], for example, collagen I, III, and V are the principal components of dermal heterotypic collagen fibrils. Of these, collagen $I$ is most abundant making up around $70 \%$. Collagen III and V comprise around $20 \%$ and $>5 \%$, respectively, of all dermal collagens [79-82]. The collagen fibrils in the papillary ECM are generally thinner than those found in the reticular ECM. Collagen fibril diameter is in part determined by the ratio of collagen III and collagen V, the latter nucleating collagen fibril formation leading to thinner fibrils [83].

Additional key regulators of collagen fibrillogenesis are proteoglycans and matricellular proteins. Decorin, a dermatan sulfate/chondroitin sulfate SLRP, and versican, a chondroitin sulfate proteoglycan, are the most abundant proteoglycans in the skin [84]. Decorin shows a higher abundance in the papillary ECM and versican in the reticular ECM $[84,85]$. The importance of these proteoglycans in the skin is reflected by skin fragility in decorin- deficient mice [86] and in dermatan sulfate-deficient humans [87]. Despite being of lower abundance, also other proteoglycans are important regulators of collagen fibrillogenesis in the dermis. These include the SLRPs biglycan, lumican and fibromodulin [88]. Proteoglycans can be present without GAG substitution as just the protein core. Furthermore, although not a protein, the GAG hyaluronan, which is distributed throughout the skin, shows its highest abundance in the papillary dermis [89].

The matricellular protein SPARC (Secreted Protein, Acidic, Rich in Cysteine), also known as BM40 or osteonectin, has its highest abundance in the papillary dermis [90]. It interacts directly with collagen I, III, and V [91] and may regulate maturation of procollagens and fibrillogenesis in the skin [92]. Periostin, another matricellular protein in the papillary ECM [93] can regulate collagen fibrillogenesis by directly interacting with collagen I [94]. It also interacts with BMP-1 evoking proteolytic activation of lysyl oxidase and subsequent collagen crosslinking [95]. During homeostasis the abundance of matricellular proteins is generally low in skin; however, after tissue damage or under pathological conditions they can be greatly increased and also involved in driving pathological processes such as fibrosis. In addition to the aforementioned matricellular proteins, these also include thrombospondin-1 and tenascin-c [12].

The deeper reticular dermal ECM is characterized by thicker collagen-containing fibrils and more prominent elastic fibers. Tropoelastin molecules are assembled and become crosslinked by lysyl oxidases to serve as the core of elastic fibers which associate with a multitude of proteins including fibrillins, fibulin 4 and 5 and latent transforming growth factor beta-binding protein 4 (LTBP4) (Figure 1) [96]. MS-based proteomic analysis of papillary and reticular dermis separated through laser microdissection illustrates that the difference in appearance of the ECM organization in the papillary and reticular dermis is not caused by an altered abundance of major structural proteins, such as fibrillar collagens [97]. Rather the differences in organization are achieved by alterations in lesser abundant ECM proteins and their effects on supramolecular assembly of ECM structures.

It should be emphasized that in proteome analyses of whole skin also endothelial, muscle, adipose, and specialized adnexal basement membranes and ECMs add to the detected proteome. In some situations, also immune cells may contribute [98]. When and to what extent will need further investigations. Furthermore, the proteome, its arrangement, and post-translational modifications (PTMs) change dynamically during development and aging [99].

\section{Quantitative mass spectrometry approaches to study the skin, its cells and ECM}

\subsection{A historical perspective - Mapping the proteome of skin and skin cells}

At the beginning of this millennium with the first draft of the human genome $[100,101]$, the golden era of MS-based proteomics began [102]. This was fueled by several parallel developments. The sequenced genome allowed the establishment of comprehensive protein databases that alleviated the 
identification of peptides and proteins by incomplete MS data; technical developments increased experimental robustness, instrument sensitivity, and sample throughput; the establishment of quantitative protocols allowed functional analyses addressing molecular mechanisms [103]. The proteomic analyses of skin and skin-derived cells nicely mirror the overall trend of MS-based proteomic studies. Amongst others, this is reflected by the number of publications: whereas in the $1990 \mathrm{~s}$ on average less than six manuscripts per year were published addressing proteomic analyses of skin-derived samples, this number increased to more than 20 in the $2000 \mathrm{~s}$. In the last three years more than 70 manuscripts per year presented data from MS-based experiments of skin specimens (using following PubMed search criteria: skin AND (keratinocyte OR fibroblast) AND (proteomics OR 'mass spectrometry')). This illustrates that MS-based proteomics has become a standard technique to study scientific questions related to skin pathophysiology.

Proteomic characterizations of skin-derived samples started with the generation of inventories (Table 2) [104]. From the very beginning comparative analyses were performed, i.e., relatively quantifying proteins from the different cell or disease states. Quantification was initially performed by densitometric analyses of polyacrylamide gel electrophoresis (PAGE) bands or spots. MS analyses were used qualitatively to identify proteins of interest. A major focus was the characterization of the epidermis and keratinocytes, pioneered by Celis and colleagues $[105,106]$. The underlying dermis and its major cell type, fibroblasts, were thought to generate merely the structural scaffold on which keratinocytes would execute their vital functions, and received considerably less attention. Keratinocyte lysates, and later also fibroblast lysates, were analyzed by two-dimensional (2D)-PAGE coupled to tryptic peptide-mass-mapping by matrix-assisted laser desorption ionization (MALDI)-MS $[106,107]$. Unbiased analyses of whole skin using the same approach followed few years later, e.g. characterizing protein abundance changes related with age [108]. Using the mouse as a model system, whole skin as well as separated epidermis and dermis analyses were performed [109]. Again, using unbiased 2D-PAGE-based proteome quantifications, protein abundance differences due to heat stress were quantified. In general, at that time 10-20 proteins were identified by MS per study. Whereas results were often confirmatory to existing knowledge, these studies were important steps in generating hypothesis-free, unbiased experimental workflows, which are the basis of current data-driven research in systems biology.
Around the turn of the millennium, 2D-PAGE in combination with peptide-mass-mapping by MALDI-MS got steadily replaced by peptide sequencing using a combination of liquidchromatography (LC) and electrospray ionization (ESI)-MS/MS analysis [102]. This led to a dramatic increase in protein identifications per MS study, from several tens of proteins to more than thousand. In one of the first studies, a comprehensive analysis of the plasma membrane proteome of human keratinocytes was performed identifying 1306 proteins [110]. Of these 866 carried Gene Ontology annotation of which ca. $60 \%$ were classified as an integral membrane or membrane-associated proteins, many of them being involved in cell adhesion nicely highlighting the physiological function of keratinocytes.

\subsection{Quantitative approaches}

In parallel to peptide sequence-based protein identification and the use of LC-MS/MS approaches as main MS-based proteomic workflow, relative quantification methods were developed for robust and accurate peptide-based protein quantifications [102]. Relative quantification approaches can be performed label-free or with the help of stable-isotope labeling allowing the relative comparison of two or more experimental states. Labels can be introduced by different means as well as on different stages of experiments. In general, metabolic, chemical, and enzymatic labeling approaches are discriminated, which label proteins or tryptic peptides [111]. Interestingly, skin-derived samples were among the first ones to be analyzed, or even used as showcase to highlight the benefits of the newly developed quantification approaches. This might either highlight the general interest in the skin as an important human organ, or the easy accessibility of skin as a model to study human organ function and cell-cell crosstalk.

In general, metabolic labeling approaches yield the most accurate quantitative data as biological samples can already be combined at the tissue or cell state. Unspecific losses due to subsequent sample handling will affect mixed samples to the same extent and thus will not influence quantification data. Various metabolites can be isotopically labeled to yield a labeled proteome. The most commonly used approaches employ ${ }^{2} \mathrm{D},{ }^{13} \mathrm{C}$, or ${ }^{15} \mathrm{~N}$ labeled amino acids, Stable Isotope Labeling by Amino Acids in Cell Culture (SILAC) being the most used variant [112]. Labeled amino acids introduce relatively few, precisely defined labeled sites per peptide which aids bioinformatic data interpretation. In the same year in which the SILAC approach using leucine labeling of $\mathrm{C} 2 \mathrm{C} 12$ and NIH 3 T3 cells was published, human skin fibroblasts were labeled with serine, leucine or tyrosine variants, and

Table 2. Non-comprehensive table of proteomics databases listing skin proteins. DB: database.

\begin{tabular}{|c|c|c|}
\hline Name of Database & Listed features & Reference/URL \\
\hline Human Protein Atlas & $\begin{array}{l}\text { Map of human proteins in cells, tissues and organs; antibody-based imaging, MS-based proteomics } \\
\text { and transcriptomics data }\end{array}$ & [170] https://www.proteinatlas.org/ \\
\hline Peptide Atlas & $\begin{array}{l}\text { Proteins and peptides identified in a largescale MS/MS experiments; MS output files and processed } \\
\text { search results }\end{array}$ & [171] http://www.peptideatlas.org/ \\
\hline PRIDE DB & $\begin{array}{l}\text { Proteins and peptides identified in a largescale MS/MS experiments; MS output files and search } \\
\text { results }\end{array}$ & [172] https://www.ebi.ac.uk/pride/ \\
\hline Proteomics DB & $\begin{array}{l}\text { Protein-centric database of quantitative MS/MS data including drug-target interactions; MS-based } \\
\text { proteomics and transcriptomics data }\end{array}$ & [173] https://www.proteomicsdb.org \\
\hline STRING DB & Known and predicted protein-protein interactions; metadata & [174] https://string-db.org \\
\hline UniProt & Protein sequence and annotation data; metadata & [175] https://www.uniprot.org/ \\
\hline
\end{tabular}


their response to radiation was analyzed by quantifying protein abundance differences as well as by identifying changes in protein phosphorylation $[113,114]$. The latter study, which employed an isotope-label-based identification of a PTM, was ahead of its time. Whereas Chen and colleagues used the isotope label in a targeted approach to aid identification of phosphorylated Ser140 of histone H2AFX after radiation, the full potential of this approach was unleashed when isotopic labeling was employed to quantify site-specific phosphorylation events on a global scale [115]. In the following years, SILAC-based quantification was employed, amongst others, to highlight that keratinocyte and fibroblast proteomes were relatively stable during cell culture preserving cell-type specific in vivo characteristics [116], and that gender, age, and skin localization only have minimal influences on the keratinocyte proteome [117].

Next to metabolic labeling, chemical labeling was employed to follow skin proteome dynamics in an unbiased fashion. The most widely used chemical tags are isobaric tags for relative and absolute quantitation (iTRAQ) [118] and tandem mass tags (TMT) [119]. As the tags are isobaric, sample complexity is not increased and quantification is performed on the MS/MS level compared to MSbased quantification of metabolic labeling strategies. Compared to the latter, isobaric chemical tags allow a higher degree of multiplexing [120]. Depending on the product up to 16 samples may be compared in a single analysis. iTRAQ labeling was used to study unconventional protein secretion of keratinocytes in response to UVB irradiation [121]. The authors could show that UVB-activated caspase-1 plays a general role in unconventional protein secretion. By comparing secretomes of cells treated with the caspase- 1 inhibitor YVAD with those of non-treated cells, 77 proteins were identified that were likely secreted in a caspase- 1 activitydependent manner. iTRAQ labeling in combination with skin fibroblasts was used to investigate possible side-effects of the HDAC inhibitor valproate in spinal muscular atrophy [122]. 2171 proteins were detected of which 1329 could be accurately quantified. While the majority of proteins appeared not affected by valproate treatment, collagen I, VI, and osteonectin were significantly reduced indicating potential side-effects on the ECM.

In the last years, label-free approaches integrating either MS or MS/MS signals as quantitative readout have gained popularity [103]. Due to newly developed data analysis algorithms and MSbased experiment designs, label-free quantifications yield robust accurate quantitative data, reducing costs of experiments, and increasing the dynamic range as well as the number of identifications. Thus, two- to three-fold differences can now be accurately quantified [123]. In so-called data-dependent acquisition (DDA)-based approaches MS signal intensities are used for quantification whereas MS/MS spectra of selected single peptides are recorded for identification. In data-independent acquisition (DIA)-based methods such as sequential window acquisition of all theoretical mass spectra (SWATH-MS) [124] entire $\mathrm{m} / \mathrm{z}$ ranges of precursor peptides are fragmented at the same time generating comprehensive fragment ion maps and intensities of fragments that belong to a given peptide are used for quantification [103]. In skin research, SWATH-MS was used amongst others to study the response of keratinocytes to cannabidiol identifying the transcriptional repressor $\mathrm{BACH} 1$ as cannabidiol target [125], to study the response of fibroblasts to redox stress characterizing collagen homeostasis as being redox sensitive [126], and to identify potential biomarkers for actinic keratosis, Bowen's Disease, and cutaneous squamous cell carcinoma using formalinfixed paraffin-embedded samples [127].

\subsection{Analyses of PTMs}

One of the unique features of MS-based proteomic approaches is the unbiased identification and quantification of PTMs. Dynamic regulation of PTMs is at the core of cellular signal transduction often resulting in changes of protein activities, and localizations being causal for observed cell phenotypes. Hence, from the very beginning, the characterization of PTMs has been a major focus of MS-based proteomic approaches [128]. As more than 200 different PTMs have been described, this review cannot be exhaustive. However, we would like to summarize the most relevant studies in skin biology.

As protein phosphorylation is a major determinant of protein activation and regulator of protein-protein interactions its analysis has received a lot of attention. MS-based proteomic approaches are the method of choice to study alterations in the phosphorylation status of proteins giving rise to the research field of phosphoproteomics [129]. As for expression proteomic experiments, initial studies addressing protein phosphorylation in skin cells were coupled to 2D-PAGE [130]. Especially the effect of irradiation on skin cells was analyzed by phosphoproteomic approaches, highlighting that skin fibroblasts respond differentially to low and high doses of ionizing radiation [131,132]. Later, phosphoproteomic approaches were coupled with elegant mechanistic studies. E.g. in mouse skin and mouse keratinocytes Polo-like Kinase 1 (Plk1) was identified to regulate keratinocyte planar cell polarity by phosphorylating the protein Celsr1, regulating its endosomal recruitment during mitosis [133]. Specific phosphorylation events were also shown to be important for skin cell differentiation: the kinase CSNK1a1 was shown to be important for keratinocyte progenitor maintenance by phosphorylating protein arginine methyltransferase 1 (PRMT1) [134]; receptor-interacting serinethreonine kinase 4 (Ripk4) was identified to phosphorylate the desmosome component plakophilin-1 (Pkp1) in mouse keratinocyte differentiation [135]. Absence of either of the two proteins led to enhanced epidermal carcinogenesis.

Whereas protein phosphorylation is a highly dynamic, reversible PTM being regulated by protein kinases and phosphatases, several (quasi) non-reversible PTMs were also shown to be important in skin biology and to be dysregulated in human diseases. Next to phosphorylation, protein oxidation is important in skin pathophysiology. UVB radiation, which induces amongst others an increase in reactive oxygen species (ROS), was shown to change protein abundances in keratinocytes and increase protein carbonylation [136]. In contrast, enzymatic protein oxidation plays an important role in physiological collagen maturation, hydroxyprolines and hydroxylysines being important for protein stability and crosslinking. It was shown by unbiased proteomic analyses of fibroblast ECM that amino acid hydroxylation as well as protein crosslinking by transglutaminases are altered on a global scale in skin fragility probably reflecting alterations in ECM stability $[47,137]$. 
A truly irreversible PTM is non-digestive proteolytic processing of proteins generating new proteoforms with potential new physiological functions. Several MS-based proteomic approaches have been designed that focus on the characterization of proteolytically processed proteins, which can be summarized as 'positional proteomics,' 'terminomics,' or 'degradomics' approaches [138]. Skin function critically depends on a tightly controlled protease network as highlighted by the high number of proteases expressed by the skin and invading immune cells and their frequently found dysregulation in disease [139]. Thus, several terminomics studies aimed to identify protease substrates in skin or skin cells. Terminal Amine Isotopic Labeling of Substrates (TAILS) was employed to characterize type I procollagen I processing by metalloproteases meprin $a$ and meprin $\beta$ [140]. iTRAQ-based TAILS was used to identify amongst others integrin alpha 6 and dermokine as new MMP10 substrates in mouse keratinocytes [141]. As MMP10 has been implicated in wound healing being highly expressed in keratinocytes at wound edges, processing of these adhesion molecules by MMP10 might promote cell migration. MS-based proteomic approaches were also employed to characterize ectodomain shedding of transmembrane proteins such as collagen XVII, characterizing neoepitopes in bullous pemphigoid [142].

\subsection{Proteomic analysis of the skin ECM}

The term ECM often means different things to different people and the definition of the ECM is ever-changing and expanding [143]. Classically the ECM referred to the structural material that surrounds cells and that can be divided into three major protein classes: collagens, glycoproteins and proteoglycans [144]. For mass spectrometrists/analytical chemists, the ECM was considered all the proteins that are found outside of the cell. Hynes and Naba came up with a clear definition that has been accepted by the different research communities: the matrisome [143,145], which is based on a bioinformatics approach to define the complete repertoire of ECM proteins.
They discriminate the 'core-matrisome' and 'matrisomeassociated proteins.' Core matrisome proteins (encoded by 278 genes in humans) have to contain minimally one out of 55 protein domains of known extracellular proteins and must not contain known intracellular or transmembrane domains, defining proteins that belong to the three ECM protein classes collagens, glycoproteins, and proteoglycans. Matrisomeassociated proteins are made up of ECM-affiliated proteins, ECM regulators, and secreted factors (encoded by 778 genes in humans). Whereas we fully support the matrisome approach, we would like to point out that from a biological point of view important information might be missed, when MS-based proteomic data are filtered on matrisome proteins without taking other proteins into account that are identified in biochemically enriched ECM samples (Figure 2). E.g. cell surface receptors interacting with ECM proteins and important for cellular phenotypes are lost in filtered data. Also, secreted lysosomal proteins, like lysosomal cathepsins, are not listed in matrisome-associated proteins.

Gel-based approaches for MS sample preparation are generally considered as old-fashioned, especially as they are not automatable and labor-intensive. Also, not all proteins are well soluble in SDS-based buffers, or well separable by PAGE, such as some membrane proteins or highly crosslinked ECM proteins found in corneocytes. Due to fixation in the gel matrix, protein recovery might also be limited; however, when sample amount is not a limiting factor, gel-based approaches still yield the most comprehensive coverage of ECM proteins according to our observations (Figure 2) (own unpublished data and [146]). Especially as crosslinked ECM proteins are often very stable and weakly soluble, gel-based approaches probably support protease accessibility and increase peptide recovery. Thus, approaches similarly as outlined in Figure 2 were performed to study the confluence switch in human keratinocytes, identifying that cell confluence inhibits plasmin via SERPINE1 activation leading to increased perlecan deposition and cell adhesion

a

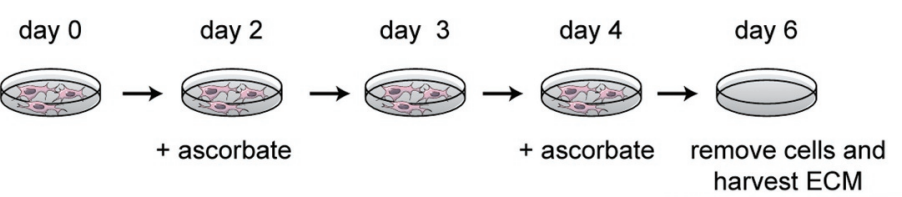

harvest ECM

b (4\% SDS, 0.1 M DTT)

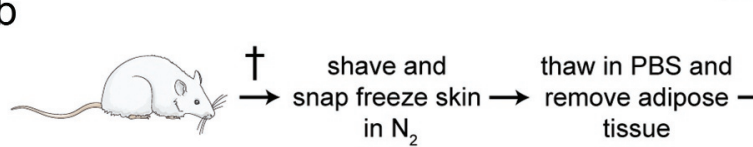

homogenize in ULTRA-TURRAX:

$\rightarrow$ boil for vortex; $20 \mathrm{~min}(4 \% \mathrm{SDS}$, $0.1 \mathrm{M}$ TRIS-HCl)

SDS-PAGE + in gel digestion OR

Gel-aided sample preparation (GASP)

$\downarrow$

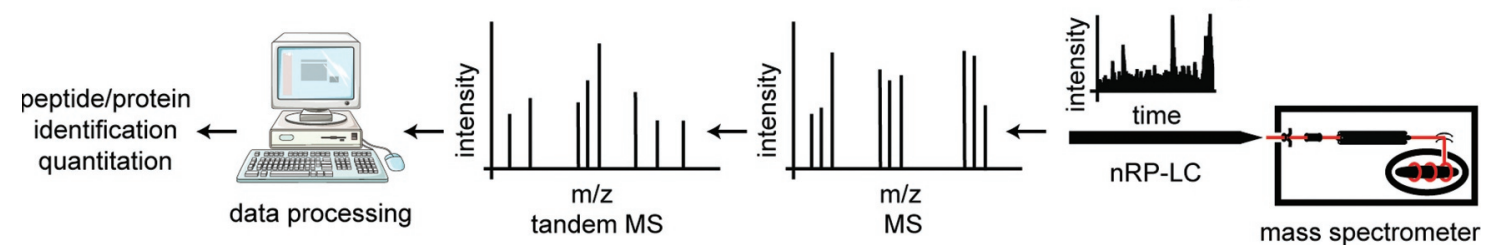

Figure 2. Bottom-up MS-based proteomic analyses of ECM and skin samples. (a) Analysis of ECM derived from primary skin cell cultures. Skin fibroblasts or keratinocytes are kept in culture for 6 days and ascorbate is added to the culture medium to support collagen hydroxylation. Cells are removed by addition of $0.5 \%$ Triton X-100 in $20 \mathrm{mM} \mathrm{NH}_{4} \mathrm{OH}$ for $30 \mathrm{~s}$ according to [176]. (b) Analysis of whole skin. After sacrifice, skin is shaved and punch biopsies are taken. Skin is macroscopically cleaned from adipose tissue and prepared as in [154]. Due to highly crosslinked ECM complexes, SDS-polyacrylamide gel-based digestion protocols [177] yield the most comprehensive coverage of ECM proteins (own-unpublished results). 
[147]. Comparing the matrisomes of mouse skin fibroblasts expressing a constitutively active variant of the transcription factor NRF2 and WT cells, it was shown that NRF2 supports cellular senescence via deposition of a senescence-promoting matrisome. The alterations in the matrisome promoted accelerated wound healing, but also tumor growth [148].

\section{Proteomic alterations in skin fragility diseases}

Illustrative examples of diseases affecting the skin ECM are genetic skin fragility disorders that manifest with blistering and easy breakability of the skin upon minor mechanical stress or shearing forces. These disorders are caused by pathogenic variants in genes encoding structural proteins of the epidermis or the DEJZ and are collectively called epidermolysis bullosa (EB). EB is a group of similar, yet distinct disorders, and mutations in more than different 20 genes are known to cause EB [149].

One EB subtype with a particularly severe and interesting phenotype is recessive dystrophic EB (RDEB). It is caused by mutations in the COL7A1 gene that encodes collagen VII, the major protein constituent of the anchoring fibrils [150] (Figure 3). Although RDEB is monogenic, i.e. mutations in a single gene

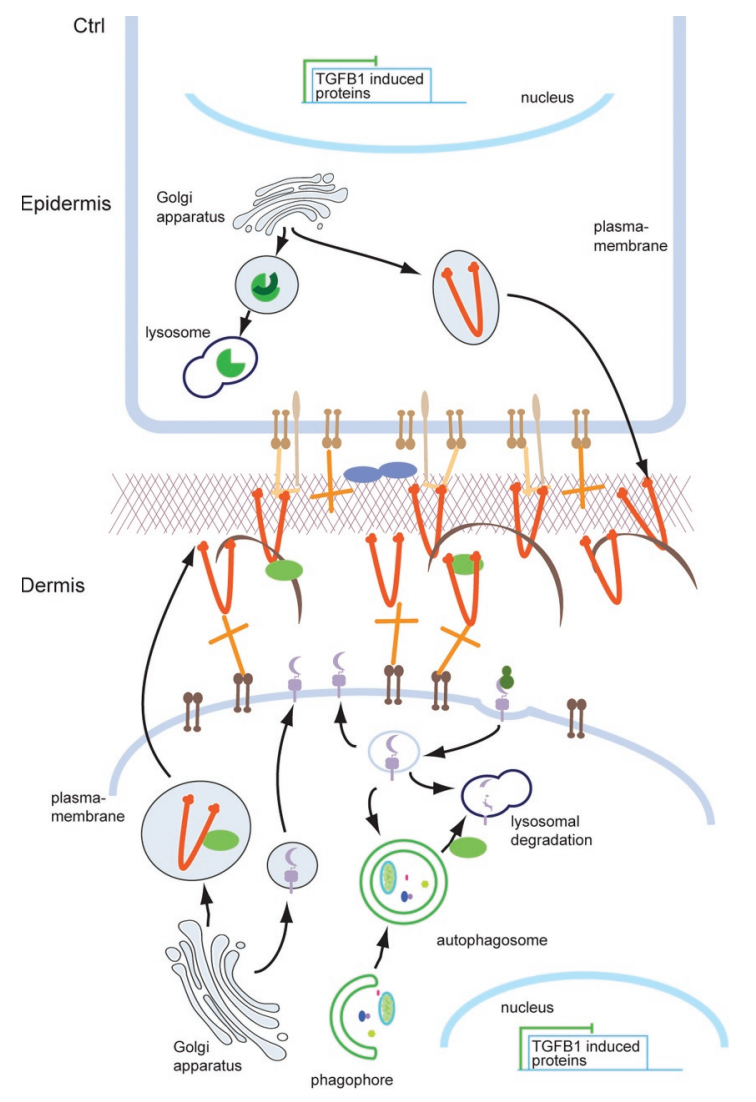

cause the disease, the consequences at tissue level are progressive and remarkably complex. Loss of collagen VII leads to lack of functional anchoring fibrils and to the separation of the epidermis from the dermis upon mechanical stress, clinically manifesting as skin blistering. But the loss of anchoring fibrils also destabilizes the dermal tissue architecture, and the blisters heal with scarring [151]. Repeated cycles of blistering and scarring become associated with generalized soft tissue fibrosis with advancing course of the disease. Although the clinical picture has been long known, the disease mechanisms remained elusive for a long time. Recent global proteomic analyses have delivered novel cues on molecular and cellular events contributing to the phenotype and on promising therapeutic targets.

A collagen VII hypomorphic mouse model of RDEB led to the identification of the fact that collagen VII-deficiency causes dysregulation of TGF-beta 1 signaling [152]. However, it was not clear how profound the alterations of the proteome in general, and the ECM in particular, are due to the loss of a single protein. In several disease proteomic studies, we could show that loss of collagen VII affects both, skin fibroblasts and keratinocytes, and has numerous cell intrinsic and extrinsic implications. The full spectrum of consequences of

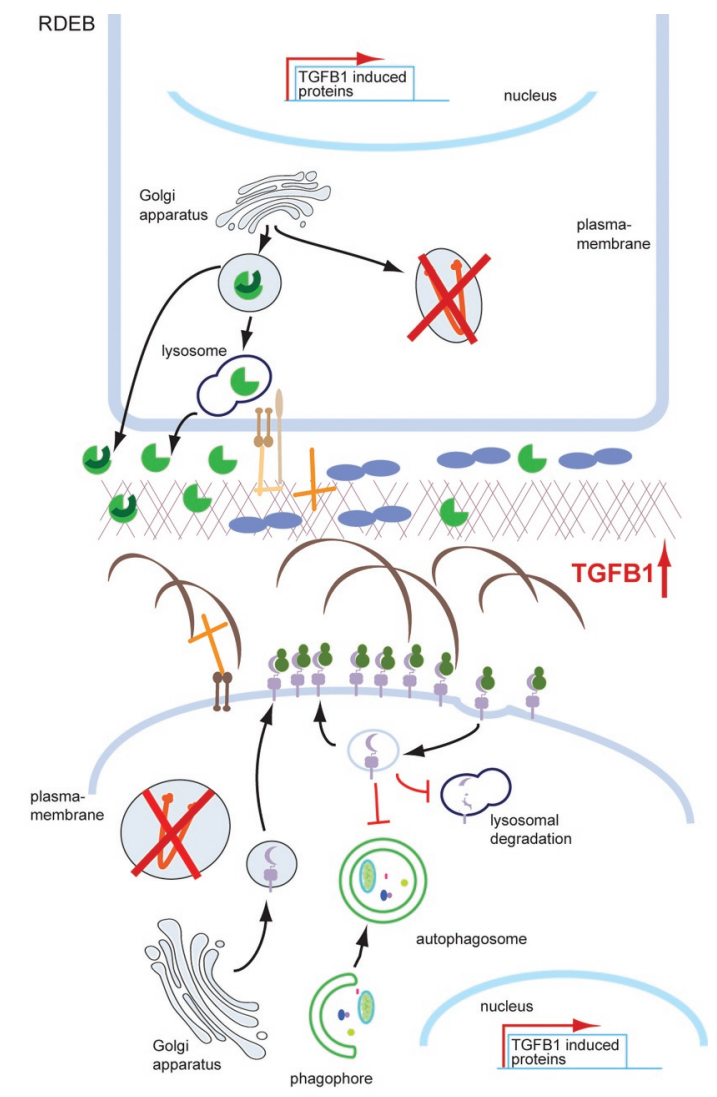

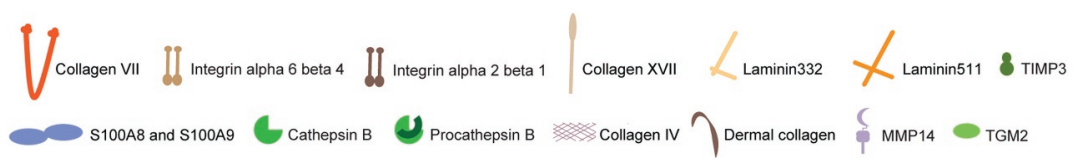

Figure 3. Dysregulation of the proteome due to loss of collagen VII in RDEB. Loss of collagen VII affects both, intra- and extracellular proteomes of skin fibroblasts and keratinocytes. Increased TGF-beta 1 signaling appears to be a driver of the disease. Fibrosis and inflammation are increased, and lysosomal capacity is decreased in RDEB. However, increased extracellular activity of lysosomal hydrolases such as cathepsin B might contribute to blister formation. Interestingly, matrix metalloproteases, e.g. MMP14 are less active indicating a disturbance of the entire protease web. 
collagen VII loss was gained from a number of omics analyses of skin cells and whole skin tissue, and the biological validation of the observed changes in vitro and in vivo.

Combinatorial omics showed that in keratinocytes loss of collagen VII leads to the downregulation of mRNA levels of several collagen VII binding partners, indicating that this loss is sensed by cells and that it affects gene transcription [48]. Furthermore, keratinocytes appear to actively contribute to the inflammation observed in RDEB individuals, amongst others by secreting S100A8 and S100A9 proteins, which together form calprotectin, a biomarker for acute and chronic inflammation [153]. Interestingly, also protein homeostasis appeared altered leading to the enhanced secretion of active lysosomal proteases from keratinocytes [48]. In fibroblasts, loss of collagen VII negatively affects the abundance and deposition of several basement membrane components such as collagen IV and laminin 511. This suggests that in vivo loss of collagen will likely weaken the suprastructure of the basement membrane and amplify the skin fragility phenotype caused by a lack of functional anchoring fibrils (Figure 3) [47]. Due to the concomitant loss of collagen VIIbinding partners, among them transglutaminase II (TGM2), dermal collagens may have fewer crosslinks negatively affecting dermis integrity [137].

Disease proteomics approaches not only contributed to understanding the molecular consequences of lack of collagen VII in RDEB, but also helped outline mechanisms of pharmacological interference with TGF-beta 1 signaling in a preclinical study employing the RDEB mouse model [154]. The small-molecule angiotensin II type 1 receptor antagonist losartan, known to negatively interfere with TGF-beta 1 signaling, caused a reduction of proteins involved in complement activation and immune and inflammatory responses in the RDEB mouse, indicating that by limiting tissue inflammation and fibrosis losartan ameliorates the disease phenotype [154]. These findings led to the initiation of a phase $1 /$ II clinical study testing the effects of losartan treatment in children with RDEB (EudraNr. 2015-003670-32), which is currently ongoing.

In line with the fact that the transcriptome of RDEB fibroblasts resembles that of cancer-associated fibroblasts [155] (Figure 3), proteomic analysis of paraffin-embedded human tissue identified similarities between the microenvironment of RDEB-associated cutaneous squamous cell carcinoma (CSCC) and sporadic metastasizing non-RDEB-CSCC, indicating that alterations in the ECM contribute to cancer invasion in RDEB [156]. Taken together, disease proteomic analyses significantly contributed to outlining the complex and widespread molecular changes underlying loss of collagen VII and contributing to RDEB pathology.

Another relevant example in which disease proteomic approaches generated new knowledge is perturbed wound healing and chronic wounds. These may occur in rare diseases, such as RDEB [151], but are also prevalent in Western life-style diseases such as diabetes and obesity, as well as with aging. Normal wound healing proceeds in a highly orchestrated manner in three phases, i) inflammation; ii) re-epithelialization of the epidermis; and iii) tissue remodeling of the regenerated dermis [63]. In slow-healing, chronic wounds these phases do not follow each other in a precisely regulated manner, but are retarded and/or derailed. The reasons are not fully understood. Several investigations have addressed re-epithelialization and epidermal regeneration [157], but only recently did disease proteomic approaches help identify changes in the dermis and define novel cell subpopulations that contribute to poor wound closure [158].

MS-based proteomics have been used since the early 2000s as an analytic tool for studying wound healing [159]. Despite the obvious necessity for wound healing of a timely and wellcoordinated deposition and arrangement of the ECM, few studies have directly by means of proteomics interrogated the ECM in physiological and pathophysiological wounds. The insights toward specific changes in the ECM that have come from general proteomic analyses of diseased skin are scarce and often limited to the description of altered abundance in individual proteins. Collagen VI was shown to be increased in human scleroderma skin [160] and the SLRP asporin in human keloids. In one of the few studies with a more direct focus on the ECM in normal and pathologically healing skin Eming et al. compared wound exudates from healing leg wounds and chronic venous leg ulcers [161]. Healing wounds showed an increased abundance of collagen I, collagen III, perlecan, COMP, lumican, and fibulin. Contrastingly, exudates from chronic wounds contained more transitional ECM proteins, fibronectin, and vitronectin. Chronic ulcers also contained a unique abundance of olfactomedin-4, an ECM protein with reported immunosuppressive activities [161,162]. In addition, chronic wounds showed an imbalance in proteolytic activities due to the dysregulated balance of proteases and protease inhibitors [161].

Analyses of wound exudates have also brought some insights to the general proteolytic microenvironment in wounds. This has been achieved by analyzing neo-N-termini performing TAILS-proteomics of wound exudates collected from acute porcine skin wounds or negative pressure-treated traumatic porcine and human wounds $[163,164]$ and led to the identification of biomarker candidates for specific stages in wound healing progression [164].

A challenge with analyzing the ECM from whole extracts of healthy and diseased skin is that aspects of differential organization and spatial distribution cannot be resolved. Toward this end, analyses of microdissected skin have been performed; this would allow a description of the spatial abundance of proteins [97]. Imaging MS may be a more useful approach to provide such information and one study showed that increased abundance of collagen XII was associated with wound beds of chronic wounds [165]. Such approaches have also been combined with tissue decellularization to improve the resolution of low abundant ECM proteins [166]. The obvious drawback with this is that more soluble ECM components can be lost and also the detergents and protocols used for decellularization will impact the ECM arrangement, thus the resulting data do not represent ECM in a native state.

Analyses of cultured fibroblasts can provide highly relevant information of pathomechanisms in skin diseases. This is due to that the proteome of cultured human fibroblasts remains rather stable over prolonged time in culture [116]. Cultured fibroblasts generally show some level of plasticity but also 
maintain organ-specific differences [167]. An advantage of using cultured cells is that the separation of deposited ECM and cells can be effectively and quickly achieved, limiting concerns of ECM degradation and reduced resolution due to contamination of cellular proteins.

Along the same lines, we recently leveraged the high-level proteome stability of culture fibroblasts to provide new insights into the pathomechanism of chronic wounds. By performing MSbased proteomic profiling of cultured dermal fibroblasts isolated from unwounded control skin, from acute healing operation wounds and from chronic wounds, combined with biological validation, we could disclose that chronic wound fibroblasts represent a specific cellular phenotype [168]. This phenotype is associated with an inability to heal wounds due to reduced motility, increased contractility, and senescence-like lysosomal dysfunction. Together, these changes make chronic woundassociated fibroblasts (cWAFs) an active contributor to wound healing failures and their targeting to eliminate or reverse their phenotype could be an attractive approach for the treatment of chronic wounds [168].

\section{Expert opinion}

MS-based proteomic approaches have significantly contributed to our understanding of skin pathophysiology. Whereas initial studies were performed to establish comprehensive inventories of skin and skin cell proteomes, subsequent quantitative approaches paved the way to functional studies addressing molecular mechanisms underlying skin disease. As inventories appear to be complete and as technical advancements allow the detection of a product of basically each protein-coding-gene, the analysis of the functions of specific proteoforms will receive more and more attention. ECM proteins appear - again - to be particularly challenging as their PTMs are not easy to analyze by MS. Thus, potential alterations in the glycosylation patterns of ECM proteins in disease settings remain truly understudied. Also, changes in protein crosslinking and oxidations are not well understood on a system level.

The necessity to study protein functions is nicely illustrated by studies in RDEB. Whereas both skin fibroblasts and keratinocytes exhibit alterations in their degradative proteome, fibroblasts expressing increased levels of MMP14 and keratinocytes of cathepsin B, only the latter appears to be active and might contribute to blister formation in RDEB. Approaches such as TAILS, directly addressing altered protease activities on a global scale promise to shed more light on skin disease mechanisms, potentially revealing new druggable targets to be tested in pre-clinical and clinical settings.

The increased sensitivity of modern LC-MS/MS systems allows the analysis of less and less cells. For skin research, this opens the opportunity to couple cell perturbations in microfluidic devices to MS-based analyses. Especially in the field of wound healing this holds great promise. E.g. the effects of surface coatings, surface tension, and rigidity on cell migration and adhesion can now be tested in screen-type of experiments [169]. By analyzing the proteomes of respective cells mechanisms actively supporting wound healing can be discerned. On the translational level this knowledge facilitates the design of novel, biologically active wound dressings.

Taken together, the increased ease of handling of LC-MS /MS systems and automated/streamlined data analyses pipelines together with increased distribution and usage of LC-MS /MS approaches will ensure that also in the coming years MSbased proteomic approaches will play a vital part in skin disease research. As in other fields, these approaches are likely moving into the clinic supporting new developments to be used in diagnostic and prognostic settings.

\section{Funding}

This work was supported by the Swiss National Science Foundation (SNSF) and the canton of Fribourg (JD), the German Research Foundation (DFG) through DE 1757/3-2 (JD), NY90/2-1, NY90/3-2, NY90/5-1 and SFB850 project $B 11(A N)$, and a research grant from the dystrophic epidermolysis bullosa research association (DEBRA) Nyström-Bruckner-Tuderman 1 (LBT, AN).

\section{Declaration of interest}

The authors have no relevant affiliations or financial involvement with any organization or entity with a financial interest in or financial conflict with the subject matter or materials discussed in the manuscript. This includes employment, consultancies, honoraria, stock ownership or options, expert testimony, grants or patents received or pending, or royalties.

\section{Reviewer disclosures}

Peer reviewers on this manuscript have no relevant financial or other relationships to disclose.

\section{References}

Papers of special note have been highlighted as either of interest $(\bullet)$ or of considerable interest $(\bullet)$ to readers.

1. Vandergriff TW. Chapter 1. Anatomy and physiology. In: Dermatology. 4th ed. 2008. Elsevier, ISBN: 9780702062759.

2. Schaefer I, Rustenbach SJ, Zimmer L, et al. Prevalence of skin diseases in a cohort of 48,665 employees in Germany. DRM. 2008;217(2):169-172.

3. lozzo RV, Gubbiotti MA. Extracellular matrix: the driving force of mammalian diseases. Matrix Biol. 2018;71-72:1-9.

4. Watt FM, Fujiwara H. Cell-extracellular matrix interactions in normal and diseased skin. Cold Spring Harb Perspect Biol. 2011;3(4). DOI:10.1101/cshperspect.a005124

5. Hynes RO. The evolution of metazoan extracellular matrix. J Cell Biol. 2012;196(6):671-679.

6. Kivirikko KI. Collagens and their abnormalities in a wide spectrum of diseases. Ann Med. 1993;25(2):113-126.

7. Rappu P, Salo AM, Myllyharju J, et al. Role of prolyl hydroxylation in the molecular interactions of collagens. Essays Biochem. 2019;63 (3):325-335.

8. Schmelzer CEH, Nagel MBM, Dziomba S, et al. Prolyl hydroxylation in elastin is not random. Biochim Biophys Acta. 2016;1860 (10):2169-2177.

9. Vadon-Le Goff S, Hulmes DJS, Moali C. BMP-1/tolloid-like proteinases synchronize matrix assembly with growth factor activation to promote morphogenesis and tissue remodeling. Matrix Biol. 2015;44-46:14-23.

10. Schuppan D, Ashfaq-Khan M, Yang AT, et al. Liver fibrosis: direct antifibrotic agents and targeted therapies. Matrix Biol. 2018;6869:435-451. 
11. Bruckner-Tuderman L, Schnyder UW, Winterhalter KH, et al. Tissue form of type VII collagen from human skin and dermal fibroblasts in culture. Eur J Biochem. 1987;165(3):607-611.

12. Nyström A, Bruckner-Tuderman L. Matrix molecules and skin biology. Semin Cell Dev Biol. 2019;89:136-146.

13. Nystrom A, Bernasconi R, Bornert O. Therapies for genetic extracellular matrix diseases of the skin. Matrix Biol. 2018;71-72:330-347.

14. Nystrom A, Bornert O, Kuhl T. Cell therapy for basement membrane-linked diseases. Matrix Biol. 2017;57-58:124-139.

15. Lechler T. Chapter 5, Growth and differentiation of the epidermis. In: Kang, Amagai, Bruckner, Enk, Margolis, McMichael, Orringer, editors. Fitzpatrick's dermatology, 9th, 2019. 2 Bände (xxvii, 3949 Seiten). ISBN10 0071837795.

16. Murphy JE, Robert C, Kupper TS. Interleukin-1 and cutaneous inflammation: a crucial link between innate and acquired immunity. J Invest Dermatol. 2000;114(3):602-608.

17. Nauroy $P$, Nyström A. Kallikreins: essential epidermal messengers for regulation of the skin microenvironment during homeostasis, repair and disease. Matrix Bio Plus. 2019;100019. DOI: 10.1016/j. mbplus.2019.100019

18. Abdayem R, Formanek F, Minondo AM, et al. Cell surface glycans in the human stratum corneum: distribution and depth-related changes. Exp Dermatol. 2016;25(11):865-871.

19. Sandjeu Y, Haftek M. Desmosealin and other components of the epidermal extracellular matrix. J Physiol Pharmacol. 2009;60(Suppl 4):23-30.

20. Dos Santos M, Michopoulou A, André-Frei V, et al. Perlecan expression influences the keratin 15-positive cell population fate in the epidermis of aging skin. Aging (Albany NY). 2016;8(4):751-768.

21. Gallo R, Kim C, Kokenyesi R, et al. Syndecans- 1 and -4 are induced during wound repair of neonatal but not fetal skin. J Invest Dermatol. 1996;107(5):676-683.

22. lozzo RV, Schaefer L. Proteoglycan form and function: A comprehensive nomenclature of proteoglycans. Matrix Biol. 2015;42:11-55.

23. Tidman MJ, Eady RA. Ultrastructural morphometry of normal human dermal-epidermal junction. The influence of age, sex, and body region on laminar and nonlaminar components. J Invest Dermatol. 1984;83(6):448-453.

24. Walko G, Castañón MJ, Wiche G. Molecular architecture and function of the hemidesmosome. Cell Tissue Res. 2015;360 (3):529-544.

25. Nishie W, Kiritsi D, Nyström A, et al. Dynamic interactions of epidermal collagen XVII with the extracellular matrix: laminin 332 as a major binding partner. Am J Pathol. 2011;179 (2):829-837.

26. Koch M, Veit G, Stricker S, et al. Expression of type XXIII collagen mRNA and protein. J Biol Chem. 2006;281(30):21546-21557.

27. Peltonen S, Hentula $M$, Hägg $P$, et al. A novel component of epidermal cell-matrix and cell-cell contacts: transmembrane protein type XIII collagen. J Invest Dermatol. 1999;113(4):635-642.

28. Veit G, Zwolanek D, Eckes B, et al. Collagen XXIII, novel ligand for integrin alpha2beta1 in the epidermis. J Biol Chem. 2011;286 (31):27804-27813.

29. Watanabe M, Natsuga K, Nishie W, et al. Type XVII collagen coordinates proliferation in the interfollicular epidermis. Elife. 2017;6. DOI:10.7554/eLife.26635.

30. Franzke C-W, Bruckner-Tuderman L, Blobel CP. Shedding of collagen XVII/BP180 in skin depends on both ADAM10 and ADAM9. J Biol Chem. 2009;284(35):23386-23396.

31. Jacków J, Löffek S, Nyström A, et al. Collagen XVII shedding suppresses re-epithelialization by directing keratinocyte migration and dampening mTOR signaling. J Invest Dermatol. 2016;136 (5):1031-1041.

32. Veit G, Zimina EP, Franzke C-W, et al. Shedding of collagen XXIII is mediated by furin and depends on the plasma membrane microenvironment. J Biol Chem. 2007;282(37):27424-27435.,

33. Te Molder L, Juksar J, Harkes R, et al. Tetraspanin CD151 and integrin alpha3beta1 contribute to the stabilization of integrin alpha6beta4-containing cell-matrix adhesions. J Cell Sci. 2019;132 (19). DOI:10.1242/jcs.235366.
34. Hohenester E. Structural biology of laminins. Essays Biochem. 2019;63(3):285-295.

35. Wegner J, Loser K, Apsite $\mathrm{G}$, et al. Laminin a5 in the keratinocyte basement membrane is required for epidermal-dermal intercommunication. Matrix Biol. 2016;56:24-41.

36. Morgner J, Ghatak S, Jakobi T, et al. Integrin-linked kinase regulates the niche of quiescent epidermal stem cells. Nat Commun. 2015;6. DOI:10.1038/ncomms9198.

37. Has C, Nyström A. Epidermal basement membrane in health and disease. Curr Top Membr. 2015;76:117-170.

38. Hamill KJ, Langbein $L$, Jones JCR, et al. Identification of a novel family of laminin N-terminal alternate splice isoforms: structural and functional characterization. J Biol Chem. 2009;284(51):35588-35596.

39. Barrera V, Troughton LD, lorio V, et al. Differential distribution of laminin N-terminus a31 across the ocular surface: implications for corneal wound repair. Invest Ophthalmol Vis Sci. 2018;59 (10):4082-4093.

40. Walko G, Castanon MJ, Wiche G. Molecular architecture and function of the hemidesmosome. Cell Tissue Res. 2015;360(3):529-544.

41. Has C, Nyström A, Saeidian AH, et al. Epidermolysis bullosa: molecular pathology of connective tissue components in the cutaneous basement membrane zone. Matrix Biol. 2018;71-72:313-329.

42. Behrens DT, Villone $D$, Koch $M$, et al. The epidermal basement membrane is a composite of separate laminin- or collagen IV-containing networks connected by aggregated perlecan, but not by nidogens. J Biol Chem. 2012;287(22):18700-18709.

43. Ninomiya $Y$, Kagawa $M$, lyama $K$, et al. Differential expression of two basement membrane collagen genes, COL4A6 and COL4A5, demonstrated by immunofluorescence staining using peptide-specific monoclonal antibodies. J Cell Biol. 1995;130(5):1219-1229.

44. Bhave G, Colon S, Ferrell N. The sulfilimine cross-link of collagen IV contributes to kidney tubular basement membrane stiffness. Am J Physiol Renal Physiol. 2017;313(3):F596-F602.

45. Fukushige T, Kanekura T, Ohuchi E, et al. Immunohistochemical studies comparing the localization of type XV collagen in normal human skin and skin tumors with that of type IV collagen. J Dermatol. 2005;32(2):74-83.

46. Karppinen S-M, Honkanen H-K, Heljasvaara R, et al. Collagens XV and XVIII show different expression and localisation in cutaneous squamous cell carcinoma: type XV appears in tumor stroma, while XVIII becomes upregulated in tumor cells and lost from microvessels. Exp Dermatol. 2016;25(5):348-354.

47. Küttner V, Mack C, Rigbolt KTG, et al., Global remodelling of cellular microenvironment due to loss of collagen VII. Mol Syst Biol. 2013;9 (1): 657.

- Comprehensive proteomic mapping of DEB cells highlighting multifaceted pertubations due to loss of collagen VII.

48. Thriene K, Grüning BA, Bornert $\mathrm{O}$, et al. Combinatorial omics analysis reveals perturbed lysosomal homeostasis in collagen VII-deficient keratinocytes. Mol Cell Proteomics. 2018;17(4):565-579.

49. Bengtsson E, Mörgelin M, Sasaki T, et al. The leucine-rich repeat protein PRELP binds perlecan and collagens and may function as a basement membrane anchor. J Biol Chem. 2002;277(17):15061-15068.

50. Velez-DelValle C, Marsch-Moreno M, Castro-Muñozledo $F$, et al. Decorin gene expression and its regulation in human keratinocytes. Biochem Biophys Res Commun. 2011;411(1):168-174.

51. Muir AM, Massoudi D, Nguyen N, et al. BMP1-like proteinases are essential to the structure and wound healing of skin. Matrix Biol. 2016;56:114-131.

52. Rattenholl A, Pappano WN, Koch $M$, et al. Proteinases of the bone morphogenetic protein-1 family convert procollagen VII to mature anchoring fibril collagen. J Biol Chem. 2002;277(29):26372-26378.

53. Veitch DP, Nokelainen P, McGowan KA, et al. Mammalian tolloid metalloproteinase, and not matrix metalloprotease 2 or membrane type 1 metalloprotease, processes laminin-5 in keratinocytes and skin. J Biol Chem. 2003;278(18):15661-15668.

54. Broder C, Arnold P, Vadon-Le Goff S, et al. Metalloproteases meprin $\alpha$ and meprin $\beta$ are $C$ - and $\mathrm{N}$-procollagen proteinases important for collagen assembly and tensile strength. Proc Natl Acad Sci USA. 2013;110(35):14219-14224. 
55. Bekhouche $M$, Colige A. The procollagen N-proteinases ADAMTS2, 3 and 14 in pathophysiology. Matrix Biol. 2015;44-46:46-53.

56. Rousselle $P$, Scoazec JY. Laminin 332 in cancer: when the extracellular matrix turns signals from cell anchorage to cell movement. Semin Cancer Biol. 2020;62:149-165.

57. Fyrand O. Studies on Fibronectin in the Skin. DRM. 1981;162 (4):220-229.

58. O'Keefe EJ, Payne RE Jr., Russell N, et al. Spreading and enhanced motility of human keratinocytes on fibronectin. J Invest Dermatol. 1985;85(2):125-130.

59. Barker $\mathrm{TH}$, Engler AJ. The provisional matrix: setting the stage for tissue repair outcomes. Matrix Biol. 2017;60-61:1-4.

60. Canty EG, Kadler KE. Procollagen trafficking, processing and fibrillogenesis. J Cell Sci. 2005;118(7):1341-1353.

61. Kinsey R, Williamson MR, Chaudhry S, et al. Fibrillin-1 microfibril deposition is dependent on fibronectin assembly. J Cell Sci. 2008;121(16):2696-2704.

62. Lynch MD, Watt FM. Fibroblast heterogeneity: implications for human disease. J Clin Invest. 2018;128(1):26-35.

63. Willenborg S, Eming SA. Cellular networks in wound healing. Science. 2018;362(6417):891-892.

64. Rognoni E, Pisco AO, Hiratsuka T, et al. Fibroblast state switching orchestrates dermal maturation and wound healing. Mol Syst Biol. 2018;14(8):e8174.

65. Mittapalli VR, Madl J, Loffek S, et al. Injury-driven stiffening of the dermis expedites skin carcinoma progression. Cancer Res. 2016;76 (4):940-951.

66. Cescon M, Gattazzo F, Chen P, et al. Collagen VI at a glance. J Cell Sci. 2015;128(19):3525-3531.

67. Martin P, Teodoro WR, Velosa APP, et al. Abnormal collagen $\mathrm{V}$ deposition in dermis correlates with skin thickening and disease activity in systemic sclerosis. Autoimmun Rev. 2012;11(11):827-835.

68. Bonod-Bidaud $C$, Roulet $M$, Hansen $U$, et al. In vivo evidence for a bridging role of a collagen $\mathrm{V}$ subtype at the epidermis-dermis interface. J Invest Dermatol. 2012;132(7):1841-1849.

69. Agarwal P, Zwolanek D, Keene DR, et al. Collagen XII and XIV, new partners of cartilage oligomeric matrix protein in the skin extracellular matrix suprastructure. J Biol Chem. 2012;287(27):22549-22559.

70. Naylor EC, Watson REB, Sherratt MJ. Molecular aspects of skin ageing. Maturitas. 2011;69(3):249-256.

71. Eckersley A, Mellody KT, Pilkington S, et al. Structural and compositional diversity of fibrillin microfibrils in human tissues. J Biol Chem. 2018;293(14):5117-5133.

72. Haynes SL, Shuttleworth CA, Kielty CM. Keratinocytes express fibrillin and assemble microfibrils: implications for dermal matrix organization. Br J Dermatol. 1997;137(1):17-23.

73. Brinckmann J, Hunzelmann N, Kahle B, et al. Enhanced fibrillin-2 expression is a general feature of wound healing and sclerosis: potential alteration of cell attachment and storage of TGF-beta. Lab Invest. 2010;90(5):739-752.

74. Schiavinato A, Keene DR, Wohl AP, et al. Targeting of EMILIN-1 and EMILIN-2 to fibrillin microfibrils facilitates their incorporation into the extracellular matrix. J Invest Dermatol. 2016;136 (6):1150-1160.,

75. Urban Z, Hucthagowder V, Schürmann N, et al. Mutations in LTBP4 cause a syndrome of impaired pulmonary, gastrointestinal, genitourinary, musculoskeletal, and dermal development. Am J Hum Genet. 2009;85(5):593-605.

76. Grässel S, Unsöld C, Schäcke $H$, et al. Collagen XVI is expressed by human dermal fibroblasts and keratinocytes and is associated with the microfibrillar apparatus in the upper papillary dermis. Matrix Biol. 1999;18(3):309-317.

77. Schalkwijk J, Zweers MC, Steijlen PM, et al. A recessive form of the ehlers-danlos syndrome caused by tenascin-X deficiency. $\mathrm{N}$ Engl J Med. 2001;345(16):1167-1175.

78. Valcourt U, Alcaraz LB, Exposito J-Y, et al. Tenascin-X: beyond the architectural function. Cell Adh Migr. 2015;9(1-2):154-165.

79. Adachi E, Hopkinson I, Hayashi T. Basement-membrane stromal relationships: interactions between collagen fibrils and the lamina densa. Int Rev Cytol. 1997;173:73-156.
80. Brinckmann J, Bodo M, Brey M, et al. Analysis of the age-related composition of human skin collagen and collagens synthesized by fibroblast culture. Arch Dermatol Res. 1994;286(7):391-395.

81. D'Hondt S, Guillemyn B, Syx D, et al. Type III collagen affects dermal and vascular collagen fibrillogenesis and tissue integrity in a mutant Col3a1 transgenic mouse model. Matrix Biol. 2018;70:72-83.

82. Wenstrup RJ, Florer JB, Brunskill EW, et al. Type V collagen controls the initiation of collagen fibril assembly. J Biol Chem. 2004;279 (51):53331-53337.

83. Sun $M$, Chen $S$, Adams $S M$, et al. Collagen $V$ is a dominant regulator of collagen fibrillogenesis: dysfunctional regulation of structure and function in a corneal-stroma-specific Col5a1-null mouse model. J Cell Sci. 2011;124(Pt 23):4096-4105.

84. Carrino DA, Sorrell JM, Caplan Al. Age-related changes in the proteoglycans of human skin. Arch Biochem Biophys. 2000;373 (1):91-101.

85. Schönherr $E$, Beavan LA, Hausser $H$, et al. Differences in decorin expression by papillary and reticular fibroblasts in vivo and in vitro. Biochem J. 1993;290(Pt 3):893-899.

86. Danielson KG, Baribault H, Holmes DF, et al. Targeted disruption of decorin leads to abnormal collagen fibril morphology and skin fragility. J Cell Biol. 1997;136(3):729-743.

87. Miyake N, Kosho T, Mizumoto S, et al. Loss-of-function mutations of CHST14 in a new type of ehlers-danlos syndrome. Hum Mutat. 2010;31(8):966-974.

88. Smith MM, Melrose J. Proteoglycans in normal and healing skin. Adv Wound Care (New Rochelle). 2015;4(3):152-173.

89. Bertheim U, Engström-Laurent A, Hofer P-A, et al. Loss of hyaluronan in the basement membrane zone of the skin correlates to the degree of stiff hands in diabetic patients. Acta Derm Venereol. 2002;82(5):329-334.

90. Hunzelmann N, Hafner M, Anders S, et al. BM-40 (osteonectin, SPARC) is expressed both in the epidermal and in the dermal compartment of adult human skin. J Invest Dermatol. 1998;110 (2):122-126.

91. Sasaki T, Hohenester E, Göhring W, et al. Crystal structure and mapping by site-directed mutagenesis of the collagen-binding epitope of an activated form of BM-40/SPARC/osteonectin. Embo J. 1998;17(6):1625-1634.

92. Rentz TJ, Poobalarahi F, Bornstein P, et al. SPARC regulates processing of procollagen $\mathrm{I}$ and collagen fibrillogenesis in dermal fibroblasts. J Biol Chem. 2007;282(30):22062-22071.

93. Murota $\mathrm{H}$, Lingli $Y$, Katayama I. Periostin in the pathogenesis of skin diseases. Cell Mol Life Sci. 2017;74(23):4321-4328.

94. Norris RA, Damon B, Mironov V, et al. Periostin regulates collagen fibrillogenesis and the biomechanical properties of connective tissues. J Cell Biochem. 2007;101(3):695-711.

95. Maruhashi T, Kii I, Saito M, et al. Interaction between periostin and BMP-1 promotes proteolytic activation of lysyl oxidase. J Biol Chem. 2010;285(17):13294-13303.

96. Uitto J, Li Q, Urban Z. The complexity of elastic fibre biogenesis in the skin - a perspective to the clinical heterogeneity of cutis laxa. Exp Dermatol. 2013;22(2):88-92.

97. Mikesh LM, Aramadhaka LR, Moskaluk C, et al. Proteomic anatomy of human skin. J Proteomics. 2013;84:190-200.

98. Etich J, Koch M, Wagener R, et al. Gene expression profiling of the extracellular matrix signature in macrophages of different activation status: relevance for skin wound healing. Int J Mol Sci. 2019;20(20):5086.

99. Shin J-W, Kwon S-H, Choi J-Y, et al. Molecular Mechanisms of Dermal Aging and Antiaging Approaches. Int J Mol Sci. 2019;20 (9):2126.

100. Lander ES, Linton LM, Birren B, et al. Initial sequencing and analysis of the human genome. Nature. 2001;409(6822):860-921.

101. Venter JC, Adams MD, Myers EW, et al. The sequence of the human genome. Science. 2001;291(5507):1304-1351.

102. Aebersold R, Mann M. Mass spectrometry-based proteomics. Nature. 2003;422(6928):198-207.

103. Aebersold R, Mann M. Mass-spectrometric exploration of proteome structure and function. Nature. 2016;537(7620):347-355. 
104. Celis JE, Cruger D, Kiil J, et al. A two-dimensional gel protein database of noncultured total normal human epidermal keratinocytes: identification of proteins strongly up-regulated in psoriatic epidermis. Electrophoresis. 1990;11(3):242-254.

105. Celis JE, Rasmussen $\mathrm{HH}$, Olsen $\mathrm{E}$, et al. The human keratinocyte two-dimensional protein database (update 1994): towards an integrated approach to the study of cell proliferation, differentiation and skin diseases. Electrophoresis. 1994;15(11):1349-1458.

106. Rasmussen $\mathrm{HH}$, Mortz $\mathrm{E}$, Mann $\mathrm{M}$, et al. Identification of transformation sensitive proteins recorded in human two-dimensional gel protein databases by mass spectrometric peptide mapping alone and in combination with microsequencing. Electrophoresis. 1994;15(3-4):406-416.

107. Sato $H$, Suzuki A, Funahashi $M$, et al. Characteristics of growth, morphology, contractility, and protein expression of fibroblasts derived from keloid. Wound Repair Regen. 1996;4(1):103-114.

108. Gromov P, Skovgaard GL, Palsdottir $H$, et al. Protein profiling of the human epidermis from the elderly reveals up-regulation of a signature of interferon-gamma-induced polypeptides that includes manganese-superoxide dismutase and the p85beta subunit of phosphatidylinositol 3-kinase. Mol Cell Proteomics. 2003;2(2):70-84.

109. Huang CM, Foster KW, DeSilva T, et al. Comparative proteomic profiling of murine skin. J Invest Dermatol. 2003;121(1):51-64.

110. Blonder J, Terunuma A, Conrads TP, et al. A proteomic characterization of the plasma membrane of human epidermis by high-throughput mass spectrometry. J Invest Dermatol. 2004;123(4):691-699.

111. Bantscheff M, Lemeer S, Savitski MM, et al. Quantitative mass spectrometry in proteomics: critical review update from 2007 to the present. Anal Bioanal Chem. 2012;404(4):939-965.

112. Ong SE, Blagoev B, Kratchmarova I, et al. Stable isotope labeling by amino acids in cell culture, SILAC, as a simple and accurate approach to expression proteomics. Mol Cell Proteomics. 2002;1(5):376-386.

113. Zhu H, Hunter TC, Pan S, et al. Residue-specific mass signatures for the efficient detection of protein modifications by mass spectrometry. Anal Chem. 2002;74(7):1687-1694.

114. Zhu H, Pan S, Gu S, et al. Amino acid residue specific stable isotope labeling for quantitative proteomics. Rapid Commun Mass Spectrom. 2002;16(22):2115-2123.

115. Olsen JV, Blagoev B, Gnad F, et al., Global, in vivo, and site-specific phosphorylation dynamics in signaling networks. Cell. 2006;127(3): 635-648.

-. Hypothesis-free phosphoproteomic study of growth factor receptor signalling elucidating signal transduction from the plasma mebrane to the nucleus.

116. Sprenger A, Kuttner V, Biniossek ML, et al. Comparative quantitation of proteome alterations induced by aging or immortalization in primary human fibroblasts and keratinocytes for clinical applications. Mol Biosyst. 2010;6(9):1579-1582.

117. Sprenger A, Weber S, Zarai M, et al. Consistency of the proteome in primary human keratinocytes with respect to gender, age, and skin localization. Mol Cell Proteomics. 2013;12(9):2509-2521.

118. Ross PL, Huang YN, Marchese JN, et al. Multiplexed protein quantitation in saccharomyces cerevisiae using amine-reactive isobaric tagging reagents. Mol Cell Proteomics. 2004;3(12):1154-1169.

119. Dayon L, Hainard A, Licker V, et al. Relative quantification of proteins in human cerebrospinal fluids by MS/MS using 6-plex isobaric tags. Anal Chem. 2008;80(8):2921-2931.

120. Christoforou AL, Lilley KS. Isobaric tagging approaches in quantitative proteomics: the ups and downs. Anal Bioanal Chem. 2012;404 (4):1029-1037.

121. Keller $M$, Ruegg $A$, Werner $S$, et al. Active caspase-1 is a regulator of unconventional protein secretion. Cell. 2008;132(5):818-831.

122. Fuller HR, Man NT, Lam le T, et al. Valproate and bone loss: iTRAQ proteomics show that valproate reduces collagens and osteonectin in SMA cells. J Proteome Res. 2010;9(8):4228-4233.

123. Cox J, Hein MY, Luber CA, et al. Accurate proteome-wide label-free quantification by delayed normalization and maximal peptide ratio extraction, termed MaxLFQ. Mol Cell Proteomics. 2014;13(9):2513-2526.

124. Gillet LC, Navarro P, Tate S, et al. Targeted data extraction of the MS/MS spectra generated by data-independent acquisition: a new concept for consistent and accurate proteome analysis. Mol Cell Proteomics. 2012;11(6):0111 016717.

125. Casares L, Garcia V, Garrido-Rodriguez M, et al. Cannabidiol induces antioxidant pathways in keratinocytes by targeting BACH1. Redox Biol. 2020;28:101321.

126. Carne NA, Bell S, Brown AP, et al. Reductive stress selectively disrupts collagen homeostasis and modifies growth factor-independent signaling through the MAPK/Akt pathway in human dermal fibroblasts. Mol Cell Proteomics. 2019;18(6):1123-1137.

127. Azimi A, Yang P, Ali M, et al. Data independent acquisition proteomic analysis can discriminate between actinic keratosis, bowen's disease, and cutaneous squamous cell carcinoma. J Invest Dermatol. 2020;140(1):212-222 e11.

128. Hennrich ML, Gavin AC. Quantitative mass spectrometry of posttranslational modifications: keys to confidence. Sci Signal. 2015;8(371):re5.

129. von Stechow L, Francavilla C, Olsen JV. Recent findings and technological advances in phosphoproteomics for cells and tissues. Expert Rev Proteomics. 2015;12(5):469-487.

130. Yamagata A, Kristensen DB, Takeda Y, et al. Mapping of phosphorylated proteins on two-dimensional polyacrylamide gels using protein phosphatase. Proteomics. 2002;2(9):1267-1276.

131. Yang F, Stenoien DL, Strittmatter EF, et al. Phosphoproteome profiling of human skin fibroblast cells in response to low- and high-dose irradiation. J Proteome Res. 2006;5(5):1252-1260.

132. Yang F, Waters KM, Miller JH, et al. Phosphoproteomics profiling of human skin fibroblast cells reveals pathways and proteins affected by low doses of ionizing radiation. PLoS One. 2010;5(11):e14152.

133. Shrestha R, Little KA, Tamayo JV, et al. Mitotic control of planar cell polarity by polo-like kinase 1. Dev Cell. 2015;33(5):522-534.

134. Bao X, Siprashvili Z, Zarnegar BJ, et al. CSNK1a1 regulates PRMT1 to maintain the progenitor state in self-renewing somatic tissue. Dev Cell. 2017;43(2):227-239 e5.

135. Lee $\mathrm{P}$, Jiang $\mathrm{S}$, Li $\mathrm{Y}$, et al. Phosphorylation of $\mathrm{Pkp} 1$ by RIPK4 regulates epidermal differentiation and skin tumorigenesis. Embo J. 2017;36(13):1963-1980.

136. Perluigi M, Di Domenico F, Blarzino C, et al. Effects of UVB-induced oxidative stress on protein expression and specific protein oxidation in normal human epithelial keratinocytes: a proteomic approach. Proteome Sci. 2010;8(1):13.

137. Kuttner V, Mack C, Gretzmeier C, et al. Loss of collagen VII is associated with reduced transglutaminase 2 abundance and activity. J Invest Dermatol. 2014;134(9):2381-2389.

138. Eckhard U, Marino G, Butler GS, et al. Positional proteomics in the era of the human proteome project on the doorstep of precision medicine. Biochimie. 2016;122:110-118.

139. de Veer SJ, Furio L, Harris JM, et al. Proteases and proteomics: cutting to the core of human skin pathologies. Proteomics Clin Appl. 2014;8(5-6):389-402

140. Broder C, Arnold P, Vadon-Le Goff S, et al. Metalloproteases meprin alpha and meprin beta are $\mathrm{C}$ - and $\mathrm{N}$-procollagen proteinases important for collagen assembly and tensile strength. Proc Natl Acad Sci U S A. 2013;110(35):14219-14224.

141. Schlage $P$, Kockmann T, Sabino $F$, et al. Matrix metalloproteinase 10 degradomics in keratinocytes and epidermal tissue identifies bioactive substrates with pleiotropic functions. Mol Cell Proteomics. 2015;14(12):3234-3246.

142. Nishie W, Lamer S, Schlosser A, et al. Ectodomain shedding generates neoepitopes on collagen XVII, the major autoantigen for bullous pemphigoid. J Immunol. 2010;185(8):4938-4947.

143. Hynes RO, Naba A. Overview of the matrisome-an inventory of extracellular matrix constituents and functions. Cold Spring Harb Perspect Biol. 2012;4(1):a004903.

144. Mecham RP (2011)

145. Naba A, Clauser KR, Hoersch S, et al., The matrisome: in silico definition and in vivo characterization by proteomics of normal and tumor extracellular matrices. Mol Cell Proteomics. 2012;11(4): M111 014647.

- Boinformatically defined inventory of ECM and ECM-associated proteins. 
146. Tolle RC, Dengjel J. Effects of the extracellular matrix on the proteome of primary skin fibroblasts. Methods Mol Biol. 1993;193-204:2019.

147. Botta A, Delteil F, Mettouchi A, et al. Confluence switch signaling regulates ECM composition and the plasmin proteolytic cascade in keratinocytes. J Cell Sci. 2012;125(18):4241-4252.

148. Hiebert $P$, Wietecha MS, Cangkrama M, et al. Nrf2-mediated fibroblast reprogramming drives cellular senescence by targeting the matrisome. Dev Cell. 2018;46(2):145-161 e10.

149. Has C, Nystrom A, Saeidian AH, et al. Epidermolysis bullosa: molecular pathology of connective tissue components in the cutaneous basement membrane zone. Matrix Biol. 2018;71-72:313-329.

150. Sakai LY, Keene DR, Morris NP, et al. Type VII collagen is a major structural component of anchoring fibrils. J Cell Biol. 1986;103 (4):1577-1586.

151. Nystrom A, Velati D, Mittapalli VR, et al. Collagen VII plays a dual role in wound healing. J Clin Invest. 2013;123(8):3498-3509.

152. Fritsch A, Loeckermann S, Kern JS, et al. A hypomorphic mouse model of dystrophic epidermolysis bullosa reveals mechanisms of disease and response to fibroblast therapy. J Clin Invest. 2008;118(5):1669-1679.

153. Shabani F, Farasat A, Mahdavi M, et al. Calprotectin (S100A8/ S100A9): a key protein between inflammation and cancer. Inflamm Res. 2018;67(10):801-812.

154. Nystrom A, Thriene K, Mittapalli V, et al. Losartan ameliorates dystrophic epidermolysis bullosa and uncovers new disease mechanisms. EMBO Mol Med. 2015;7(9):1211-1228. \$.

. Proteomic analysis of losartan treated DEB mouse model allowed for deliniation of the drug's therapeutic actions in DEB which included reducing TGFb activity and inflammation.

155. Ng YZ, Pourreyron C, Salas-Alanis JC, et al. Fibroblast-derived dermal matrix drives development of aggressive cutaneous squamous cell carcinoma in patients with recessive dystrophic epidermolysis bullosa. Cancer Res. 2012;72(14):3522-3534.

156. Foll MC, Fahrner M, Gretzmeier C, et al. Identification of tissue damage, extracellular matrix remodeling and bacterial challenge as common mechanisms associated with high-risk cutaneous squamous cell carcinomas. Matrix Biol. 2018;66:1-21.

157. Dekoninck S, Blanpain C. Stem cell dynamics, migration and plasticity during wound healing. Nat Cell Biol. 2019;21(1):18-24.

158. Driskell RR, Lichtenberger BM, Hoste $E$, et al., Distinct fibroblast lineages determine dermal architecture in skin development and repair. Nature. 2013;504(7479): 277-281.

-. Definition of fibroblast lineages and characterization of their roles in skin biology.

159. Nanney LB, Caldwell RL, Pollins AC, et al. Novel approaches for understanding the mechanisms of wound repair. J Investig Dermatol Symp Proc. 2006;11(1):132-139.

160. Aden N, Shiwen X, Aden D, et al. Proteomic analysis of scleroderma lesional skin reveals activated wound healing phenotype of epidermal cell layer. Rheumatology (Oxford). 2008;47 (12):1754-1760.

161. Eming SA, Koch M, Krieger A, et al., Differential proteomic analysis distinguishes tissue repair biomarker signatures in wound exudates obtained from normal healing and chronic wounds. J Proteome Res. 2010;9(9): 4758-4766.
- One of the first proteomic studies on a wound healing pathology employing human samples.

162. Liu W, Rodgers GP. Olfactomedin 4 expression and functions in innate immunity, inflammation, and cancer. Cancer Metastasis Rev. 2016;35(2):201-212.

163. Sabino F, Hermes O, Egli FE, et al. In vivo assessment of protease dynamics in cutaneous wound healing by degradomics analysis of porcine wound exudates. Mol Cell Proteomics. 2015;14 (2):354-370.

164. Sabino F, Egli FE, Savickas S, et al. Comparative degradomics of porcine and human wound exudates unravels biomarker candidates for assessment of wound healing progression in trauma patients. J Invest Dermatol. 2018;138(2):413-422.

165. Taverna D, Pollins AC, Sindona G, et al. Imaging mass spectrometry for assessing cutaneous wound healing: analysis of pressure ulcers. J Proteome Res. 2015;14(2):986-996.

166. Gessel M, Spraggins JM, Voziyan P, et al. Decellularization of intact tissue enables MALDI imaging mass spectrometry analysis of the extracellular matrix. J Mass Spectrom. 2015;50(11):1288-1293.

167. Slany A, Meshcheryakova A, Beer A, et al. Plasticity of fibroblasts demonstrated by tissue-specific and function-related proteome profiling. Clin Proteomics. 2014;11(1):41.

168. Berberich B, Thriene K, Gretzmeier C, et al. Proteomic profiling of fibroblasts isolated from chronic wounds identifies disease-relevant signaling pathways. J Invest Dermatol. 2020. in press. DOI:10.1016/ j.jid.2020.02.040

169. Um E, Oh JM, Granick S, et al. Cell migration in microengineered tumor environments. Lab Chip. 2017;17(24):4171-4185.

170. Uhlen $M$, Zhang $C$, Lee $S$, et al. A pathology atlas of the human cancer transcriptome. Science. 2017;357(6352). DOI:10.1126/ science.aan2507.

171. Schwenk JM, Omenn GS, Sun Z, et al. The human plasma proteome draft of 2017: building on the human plasma peptideatlas from mass spectrometry and complementary assays. J Proteome Res. 2017;16(12):4299-4310.

172. Perez-Riverol Y, Csordas A, Bai J, et al. The PRIDE database and related tools and resources in 2019: improving support for quantification data. Nucleic Acids Res. 2019:47(D1):D442-D450.

173. Samaras $P$, Schmidt T, Frejno $M$, et al. ProteomicsDB: a multi-omics and multi-organism resource for life science research. Nucleic Acids Res. 2020;48(D1):D1153-D1163.

174. Szklarczyk D, Gable AL, Lyon D, et al. STRING v11: protein-protein association networks with increased coverage, supporting functional discovery in genome-wide experimental datasets. Nucleic Acids Res. 2019;47(D1):D607-D613.

175. UniProt C. UniProt: a worldwide hub of protein knowledge. Nucleic Acids Res. 2019;47(D1):D506-D515.

176. Vlodavsky I. Preparation of extracellular matrices produced by cultured corneal endothelial and PF-HR9 endodermal cells. Curr Protoc Cell Biol. 1999;1(1):10.4.1-10.4.14.

177. Fischer R, Kessler BM. Gel-aided sample preparation (GASP)-a simplified method for gel-assisted proteomic sample generation from protein extracts and intact cells. Proteomics. 2015;15 (7):1224-1229. 